\title{
Electron beam halo monitor for a compact x-ray free-electron laser
}

\author{
Hideki Aoyagi, ${ }^{1}$ Yoshihiro Asano, ${ }^{2}$ Toshiro Itoga, ${ }^{1}$ Nobuteru Nariyama, ${ }^{1}$ Teruhiko Bizen, ${ }^{1}$ Kenji Fukami, ${ }^{1}$ \\ Tsuyoshi Aoki, ${ }^{1}$ Shinsuke Suzuki, ${ }^{1}$ Mitsuhiro Yamaga, ${ }^{1}$ Takuya Otake, ${ }^{2}$ Takashi Tanaka, ${ }^{2}$ and Hideo Kitamura ${ }^{2}$ \\ ${ }^{1}$ Japan Synchrotron Radiation Research Institute (JASRI), 1-1-1, Kouto, Sayo-cho, Sayo-gun, Hyogo 679-5198 Japan \\ ${ }^{2}$ RIKEN SPring-8 Center, 1-1-1 Kouto, Sayo-cho, Sayo-gun, Hyogo 679-5148, Japan
}

(Received 26 November 2012; published 19 March 2013)

\begin{abstract}
An electron beam halo monitor using diamond-based detectors, which are operated in the ionization mode, has been developed for the SPring-8 Angstrom compact free-electron laser (SACLA) to protect its undulator magnets from radiation damage. Diamond-based detectors are inserted in a beam duct to measure the intensity of the beam halo directly. To suppress the degradation of the electron beam due to the installation of the beam halo monitor, rf fingers with aluminum windows are newly employed. We evaluated the effect of radiation from the Al windows on the output signal both experimentally and by simulation. The operational results of the beam halo monitor employed in SACLA are presented.
\end{abstract}

DOI: 10.1103/PhysRevSTAB.16.032802

\section{INTRODUCTION}

In high-energy particle accelerators, unintended beam loss results not only in radio activation of the apparatus but also in radiation damage to it. In particular, in x-ray freeelectron laser (XFEL) facilities and synchrotron radiation facilities, where undulators are used, even if a small part of the halo of the electron beam is continuously irradiated to permanent magnets of the undulator, the magnets may be demagnetized, resulting in a fatal problem [1]. Therefore, the electron beam halo must be monitored continuously. This is particularly important in linear accelerators, such as XFEL facilities, because the halo generated in the injector section propagates downstream.

Diamond-based detectors, which operate in the ionization mode, possess high radiation hardness [2], high heat resistance, high insulation resistance, and so forth [3]. Demonstrations of the observation of charged particles using a diamond-based detector have already been carried out at some facilities [3], and such detectors have been used operationally at the Large Hadron Collider at CERN for a wide variety of beam instruments, such as particle counters, phase monitors, beam loss monitors, and spectrometers [4,5].

We have designed and fabricated a prototype beam halo monitor using diamond-based detectors made of chemical vapor deposition diamond [6] to protect the SPring-8 Angstrom compact free-electron laser (SACLA) undulators [7]. Pulse mode measurement is adopted to avoid the background noise efficiently, since $1 \mathrm{fC} /$ pulse with $10 \mathrm{~Hz}$, for example, corresponds to $10 \mathrm{fA}$ in current mode measurement, which could be buried under the background

Published by the American Physical Society under the terms of the Creative Commons Attribution 3.0 License. Further distribution of this work must maintain attribution to the author(s) and the published article's title, journal citation, and DOI.
PACS numbers: 41.85.Ew, 29.40.Wk, 81.05.ug, 41.60.Cr

noise. The detectors are directly inserted in the beam duct to measure the intensity of the beam halo. The two diamond-based detectors mounted in the prototype are arranged to measure the halo that passes over the upper and lower sides of the core of the electron beam (Fig. 1). Evaluation tests have been performed at the beam dump of the $8 \mathrm{GeV}$ SPring- 8 booster synchrotron and at the $250 \mathrm{MeV}$ SPring-8 compact SASE source (SCSS) test accelerator, which is an extreme ultraviolet (EUV)-FEL facility. The output charge of each diamond-based detector is proportional to the number of incident electrons per pulse in the range of $2 \times 10^{3}$ to $10^{7}$ electrons/pulse. The output pulse signal is unipolar and has a full width at half maximum (FWHM) length of $0.33 \mathrm{~ns}$. The effect of the induction current of the incident electrons on the output unipolar signal can be controlled using low-pass filters (LPFs). There was no effect on EUV-FEL lasing even when the diamond-based detectors were placed at less than $1 \mathrm{~mm}$ from the beam axis. We conclude that the electron beam halo monitor is feasible for use as an interlock sensor to protect undulator permanent magnets in SACLA from radiation damage. It is to be stressed that the beam halo monitor is noninvasive and can work continuously without perturbing SASE operation. It is extremely sensitive, and the lower detection limit of $2 \times 10^{3}$ electrons/pulse corresponds to $0.3 \mathrm{fC}$ detection capability, which cannot be obtained with the other beam loss monitors, such as optical fibers and photomultipliers. The typical lower detection limit of the fiber-based Cerenkov beam loss monitor, for example, is $0.5 \mathrm{pC}$ [8]. In addition, the beam halo monitor can also be used as a diagnostic tool to get transverse profile of the beam halo.

There is a possibility that the electron beam quality will degrade when the detectors approach the core of the electron beam. In the $250 \mathrm{MeV}$ SCSS (lower energy), the beam is much more affected by wakefields than at the $8 \mathrm{GeV}$ SACLA (higher energy). The bunch length of 30-40 fs 


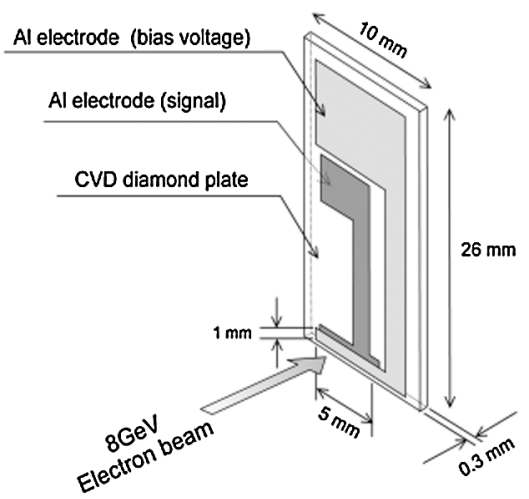

(a)

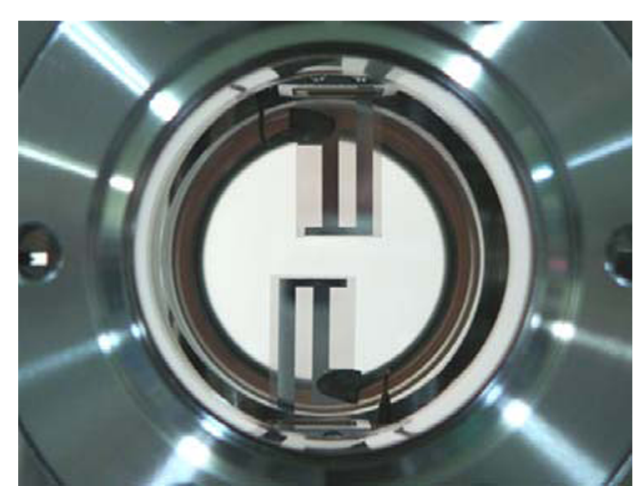

(b)

FIG. 1. Diamond-based detector and configuration of the prototype beam halo monitor. (a) The active area is the bottom part of the diamond crystal. The size of the active area is $1 \mathrm{~mm}(\mathrm{H}) \times 5 \mathrm{~mm}(\mathrm{~W})$, and the thickness is $0.3 \mathrm{~mm}$, which is sandwiched between electrodes on the both sides of the diamond crystal. (b) Two detectors set on the upper and lower sides of the beam axis. The beam core passes between them. Each detector can be actuated independently. The beam direction in (b) is from back to front.

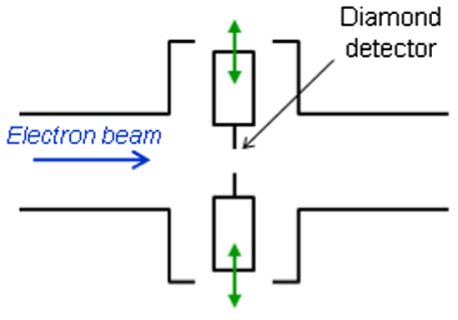

(a) Type 0

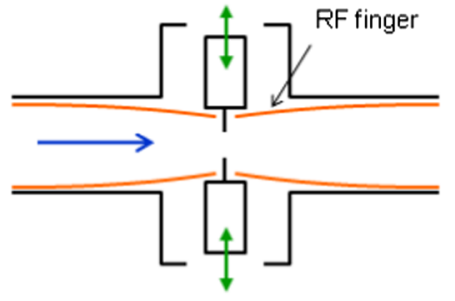

(b) Type 1

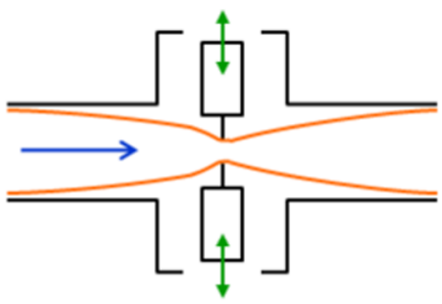

(c) Type 2

FIG. 2. Configurations of the rf fingers: (a) type 0, without fingers; (b) type 1, with fingers (the active areas of the detectors are not covered); (c) type 2, with fingers (fully covered).

(FWHM) in SACLA, however, is much shorter than 300$700 \mathrm{fs}$ (FWHM) in SCSS. Ultrashort bunches can lead to resistive wakes which mean that all electrons see a similar drop of energy. Since the XFEL lasing condition in SACLA could be severer than the EUV-FEL lasing condition in SCSS, additional devices that decrease the impedance to the beam are required. Therefore, we attempted to cover the detectors with rf fingers to completely exclude this factor, which deteriorates the stability of the XFEL lasing in SACLA, and we adopted these rf fingers in the actual monitor for SACLA $[9,10]$. The main purpose of the rf fingers is to remove the wakefield to preserve the electron beam quality. We found that, as a by-product, highfrequency components that emerge in the signal of the diamond-based detectors can be reduced in intensity.

\section{RF FINGER CONFIGURATION}

The wakefield that originates from the core of the beam generates high-frequency components in a unipolar pulse signal when the detectors are inserted in the beam duct. This component may obstruct the XFEL lasing and also increase the noise level of the output signals of the detectors. The diamond-based detectors of the prototype have no fingers (type 0 configuration), as shown in Figs. 1(b) and 2(a); thus, the high-frequency components can easily emerge in the output signal. To remove the highfrequency components, we designed two rf finger configurations [8,9]. Beryllium copper alloy $(\mathrm{BeCu})$ with the typical thickness of $0.1-0.3 \mathrm{~mm}$ is recommended as a base material of the rf fingers. In the type 1 configuration, as shown in Fig. 2(b), the rf fingers are designed to flatten the interspaces around the detector holders, but the active areas of the diamond-based detectors project between the rf fingers to measure the beam halo directly. In the type 2 configuration, as shown in Fig. 2(c), the rf fingers are designed to cover the detectors fully to connect the inner wall of the beam duct continuously and to prevent the direct irradiation of the intense wakefield from the ultrashort pulses of SACLA to the diamond-based detectors.

\section{A. Evaluation of wakefield reduction}

We evaluated the effects of the type 1 and type 2 configurations of $r f$ fingers on reducing the wakefield in the detector signal $[9,10]$. Measurements have been carried out at the $250 \mathrm{MeV}$ SCSS test accelerator. The beam size of the 


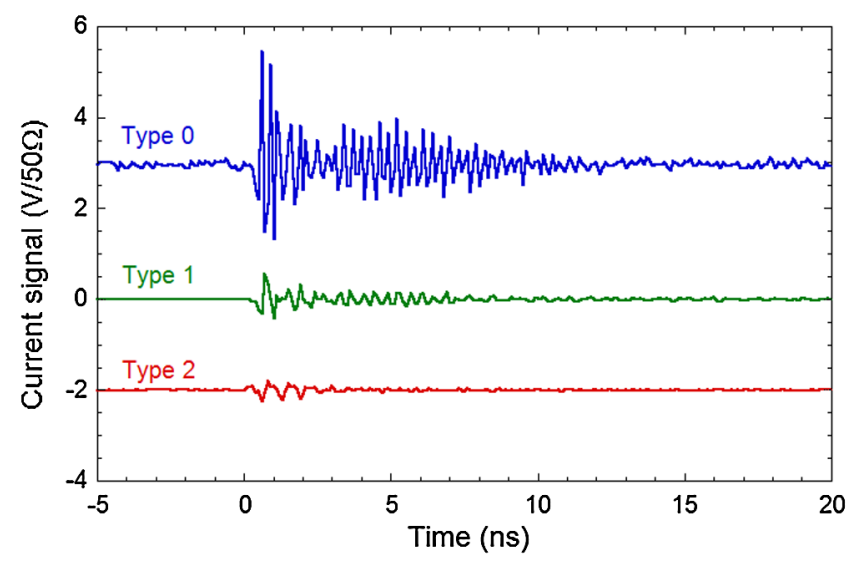

FIG. 3. Effect of configuration on reducing the wakefield.

electron beam is $0.25 \mathrm{~mm}$ (standard deviation, $\sigma$ ) and a pulse length is $300-700$ fs (FWHM). Figure 3 shows the output current signal with the high-frequency components resulting from the wakefield generated when the beam core passes between the diamond detectors. The current signal in the vertical axis is normalized to that for an electron beam charge of $0.3 \mathrm{nC} /$ pulse. The distance between each diamond-based detector and the beam core is $3.5 \mathrm{~mm}$. The bias voltage of the detector is $+100 \mathrm{~V}$. In the type 0 configuration (no rf fingers), large high-frequency components, which are caused by the induction current, can be seen. The maximum peak-to-peak amplitude is about $4[\mathrm{~V} / 50 \Omega]$. The duration of the ringing structure is about $10 \mathrm{~ns}$. The induced wake spreads in the vacuum chamber of the monitor and undergoes repeated reflection. In the case of the type 1 configuration, the amplitude is reduced markedly. This is because the interspaces around the detectors are shielded by the rf fingers. In the type 2 configuration, the duration is shortened markedly, and the high-frequency components are reduced further by covering the detectors fully with the rf fingers.

Figure 4 shows the result of the fast-Fourier-transform (FFT) analysis of the current signal. In the type 0 configuration, several peaks can be seen because the vacuum

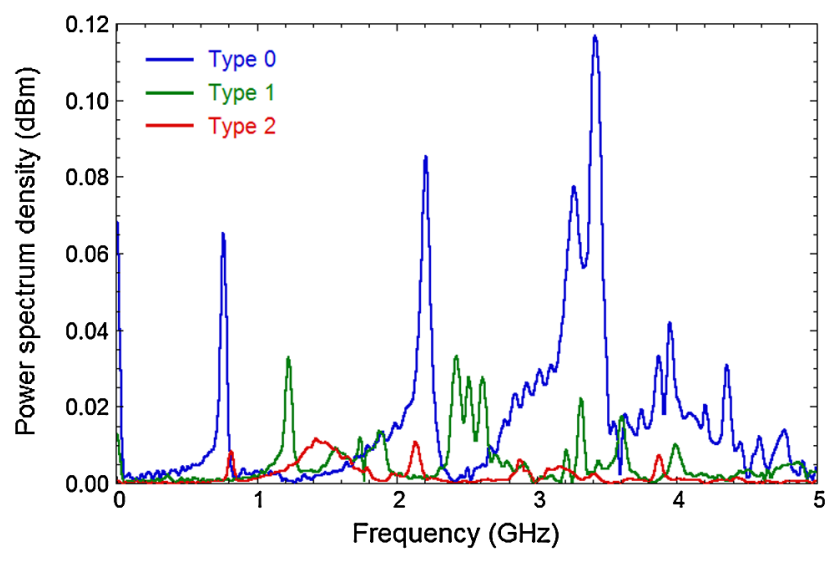

FIG. 4. FFT analysis of current signals.

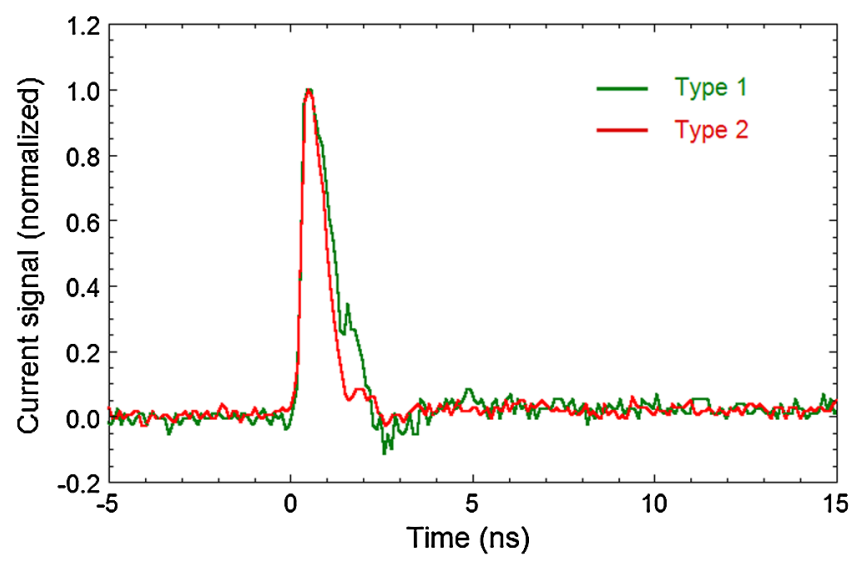

FIG. 5. Pulse shape without effect of the wakefield. The pulse heights are normalized by the peak.

chamber of the monitor has its own resonant frequencies. It can be clearly seen that the high-frequency components are decreased for the type 1 and type 2 configurations. High-frequency components with a bandwidth of $2 \mathrm{GHz}$ or wider are reduced effectively, particularly in the type 2 configuration. Therefore the high-frequency components can be easily filtered by LPFs in the type 2 configuration.

We observed the pulse shape of the original signal of the diamond-based detectors, which originated from ionization by induced electrons. These measurements were carried out under the condition that the effect of the wakefield is removed by inserting an optical transition radiation (OTR) screen upstream of the monitor to diffuse the beam core. It was confirmed with the downstream OTR screen that the beam core spreads into the whole beam duct. The unipolar pulse signal can be seen clearly even if the diamond-based detectors are covered fully with the $\mathrm{rf}$ fingers (type 2 configuration), as shown in Fig. 5. We conclude that the original signal of the diamond-based detectors is not affected by covering the detectors. Furthermore, a shoulder at a time of approximately $2 \mathrm{~ns}$ in the type 1 configuration is significantly reduced in the type 2 configuration. The shoulder is originated from a reflection at a ready-made subminiature type A (SMA) feedthrough connector for a bias voltage cable. This is because high-frequency SMA feedthrough connectors are used in the type 2 configuration to avoid the reflection.

Using the rf finger structure with the type 2 configuration, we succeeded in reducing the wakefield generated by the resonance in the vacuum chamber of the monitor without any intrinsic drawbacks. It is, rather, an advantage at the point of view of the signal processing that the highfrequency component was reduced.

\section{B. Evaluation of radiation from rf fingers}

Beryllium copper alloy $(\mathrm{BeCu})$, which has good spring characteristics, is usually used for rf fingers. However, $\mathrm{BeCu}$ has a high atomic number. Thus, if the rf fingers are placed in front of the detectors, secondary electrons and 


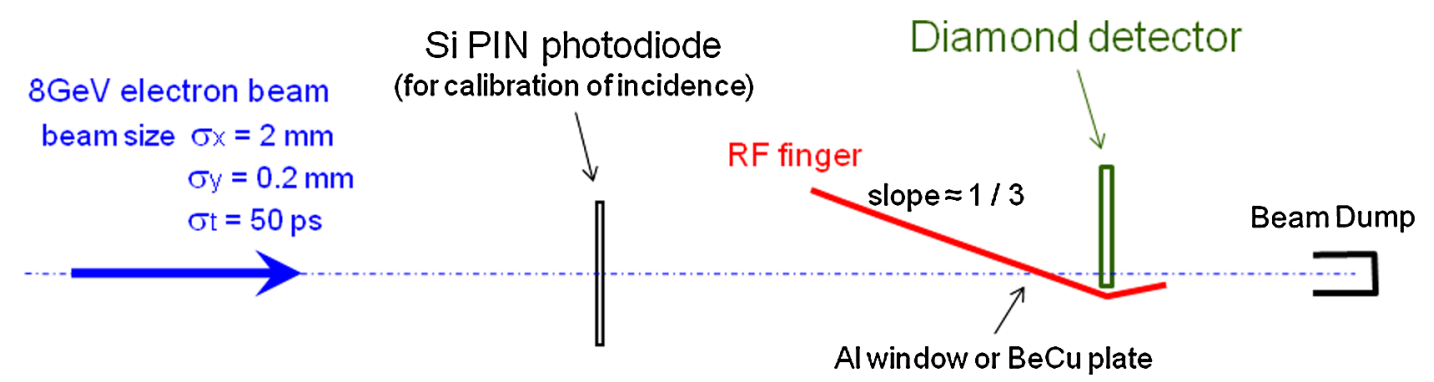

FIG. 6. Experimental setup to evaluate the effect of radiation.

bremsstrahlung will radiate from the rf fingers and the output signal of the detectors will be contaminated by them. Beam tests have been carried out at the beam dump of the $8 \mathrm{GeV}$ SPring- 8 booster synchrotron. The effect of radiation on the output signal of the diamondbased detectors was measured, and the results were compared with the simulation results [10].

The experimental setup is shown in Fig. 6. An rf finger is placed as a radiator in front of a diamond-based detector, and the inclination of the finger is about $1 / 3$, which is the inclination in actual use. This means that the effective path thickness is tripled. We measured not only $\mathrm{BeCu}$ plates but also an aluminum (Al) plate. The $\mathrm{Al}$ plate has a low atomic number and can mitigate the effect of radiation, making it a good candidate window material.

The electron beam has an energy of $8 \mathrm{GeV}$, a beam size of $2 \mathrm{~mm}$ by $0.2 \mathrm{~mm}(\sigma)$, and a pulse length of $50 \mathrm{ps}(\sigma)$. The charge of the beam was changed from $3 \times 10^{3}$ to $2 \times 10^{6}$ electrons/pulse for each condition. A silicon p-intrinsic-n (PIN) photodiode used for the calibration of incident electrons was placed upstream. The increase in intensity of the signal of the diamond-based detector due to the irradiation of the silicon PIN photodiode was practically negligible. However, when the photodiode was placed downstream of the diamond-based detector and $\mathrm{rf}$

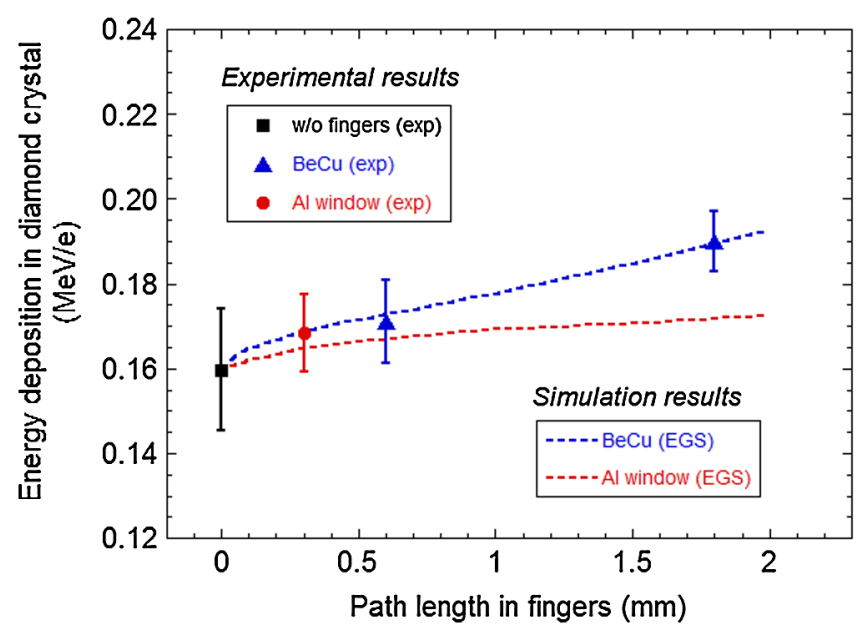

FIG. 7. Experimental and simulation results. The error bar for each experimental result indicates a measurement error of 1 standard deviation. finger, it became impossible to calibrate the incident electrons because of the unknown radiation from the rf finger.

The experimental results are shown in Fig. 7 with the simulation results. The vertical axis is the energy deposition within the diamond crystal $(t=0.3 \mathrm{~mm})$, and the horizontal axis is the effective path length of the incidence across the finger material. The dots indicate the experimental results. In the experiments, the energy depositions are converted from the output signal of the diamond-based detector.

The Electron-Gamma Shower, version 5 (EGS5) computer code system [11] was used for the simulation. EGS5 is a general-purpose package for the Monte Carlo simulation of coupled electron-photon transport in an arbitrary geometry for particles with energies from a few $\mathrm{keV}$ up to several TeV. The dashed lines in Fig. 7 indicate the simulation results.

The value of each measured energy deposition is normalized so that the measurement value without the rf finger (black square) corresponds to an energy deposition of $0.16 \mathrm{MeV} / \mathrm{e}$, which is the simulation result without the $\mathrm{rf}$ finger (a path length of $0 \mathrm{~mm}$ ). The experimental and simulation results are in good agreement within measurement errors. From the simulation results, it can be seen that the energy deposition in the diamond crystal increases to some degree as the effective path length increases, and that the energy deposition originating from the radiation inside the $\mathrm{Al}$ window is less than that originating from the $\mathrm{BeCu}$ plate. The experimental result also suggests this behavior. However, note that the increase in the energy deposition from these materials is not significant. In the case of the $\mathrm{Al}$ window with an effective path length of $0.3 \mathrm{~mm}$, which corresponds to an actual thickness of $0.1 \mathrm{~mm}$, the increase in the energy deposition is only a few percent. Therefore, we decided to adopt the rf finger structure with the type 2 configuration and $\mathrm{Al}$ windows with a thickness of $0.1 \mathrm{~mm}$ in front of the active areas of the diamond-based detectors.

\section{MECHANICAL DESIGN OF BEAM HALO MONITOR}

\section{A. rf fingers with aluminum windows}

A schematic view of the rf fingers with the $\mathrm{Al}$ windows [10] is shown in Fig. 8. The thickness of the $\mathrm{Al}$ windows is 


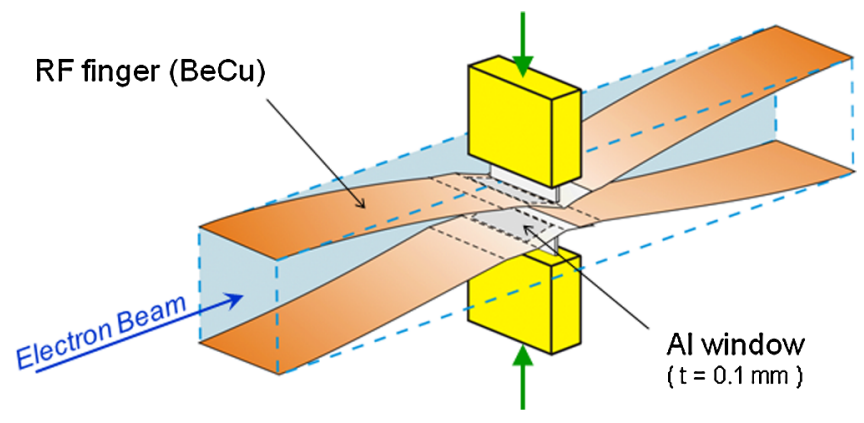

FIG. 8. Schematic view of the rf fingers with aluminum windows.

$0.1 \mathrm{~mm}$ to minimize the effect of radiation. The base material of the rf fingers is $\mathrm{BeCu}(t=0.1 \mathrm{~mm})$. To form the $\mathrm{Al}$ windows, plates of $\mathrm{Al}$ are attached to the frame of the $\mathrm{BeCu}$ fingers by spot welding. Four rf fingers are used to cover the pair of diamond-based detectors. Only the fingers on the upstream side have $\mathrm{Al}$ windows. The outside edges of four fingers are fixed on the top and bottom walls of the beam duct, which have a square cross section. The inside edges overlap each other and undergo sliding motion immediately after the diamond-based detectors, so that each detector can be actuated independently. There are two modes of operation for the beam halo monitor. In the active mode, the rf fingers are pushed inward by metal fittings, which are attached beside the detectors, to measure the beam halo close to the beam core [Fig. 9(a)]. In the shelter mode, the diamond detectors are pulled fully outward, and the size of the aperture of the beam duct is maximized [Fig. 9(b)].

\section{B. Other rf fingers}

To visually check that the diamond-based detectors and rf fingers are operating normally, a viewport is added beside the detectors, although it is desirable for the cross section of the beam duct to be square. To prevent the impedance to the beam at the viewport from increasing, a screen shield made of $\mathrm{BeCu}$ [9] is attached along the inner wall of the beam duct (Fig. 10). The screen shield has many (a)

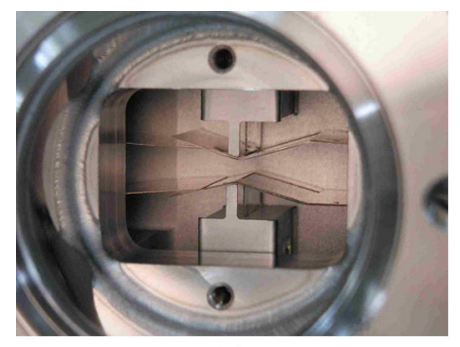

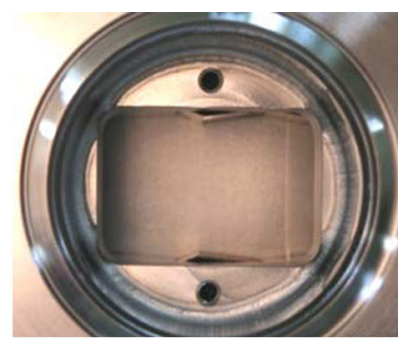

(b)
FIG. 9. Photograph of the rf fingers with $\mathrm{Al}$ windows in (a) active mode and (b) shelter mode. The beam direction is from right to left.

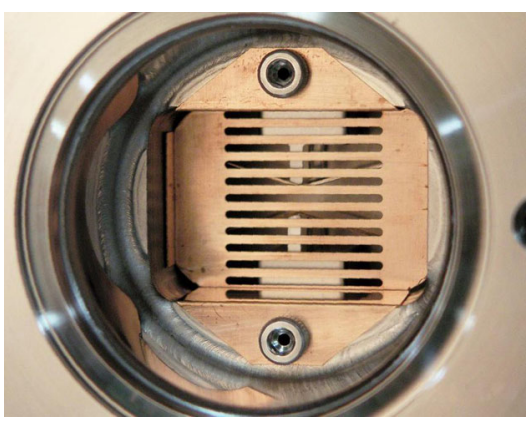

FIG. 10. Photograph of the screen shield for a viewport.

slits parallel to the beam direction; therefore, the wakefield does not penetrate the viewport while maintaining sufficient visibility.

The cross section of the beam duct inside the monitor is square, and that of the beam pipe connected to the monitor is circular. If the inner wall of the beam duct is connected with a discontinuity, the junction causes the reflection of the wakefield. We devised a beam pipe adaptor [9] with a circular cross section on one side and a square cross section on the other side, as shown in Fig. 11. The circular cross section is smoothly and continuously converted into the square cross section inside the beam pipe adaptor. The diameter of the circular cross section is $22 \mathrm{~mm}$, which is the same as that of the standard beam pipe in SACLA. The length of the square cross section is also $22 \mathrm{~mm}$; the actual width of a finger is $20 \mathrm{~mm}$. The resonance generated in the beam duct inside the monitor can be reduced using this beam pipe adaptor.

The beam adaptors are connected to the vacuum chamber of the monitor through ICF70 flanges. If they are connected using the standard ICF70 flanges, the gaps where gaskets are inserted may reach $4-5 \mathrm{~mm}$. Thus, we designed flange gap fingers made of $\mathrm{BeCu}$ [9]. Flange gap fingers are attached on both sides of the monitor, as shown in Fig. 12. The four wings of each flange gap finger touch each of the four sides of the beam pipe adaptors, and the inner wall of the beam duct is linked continuously.

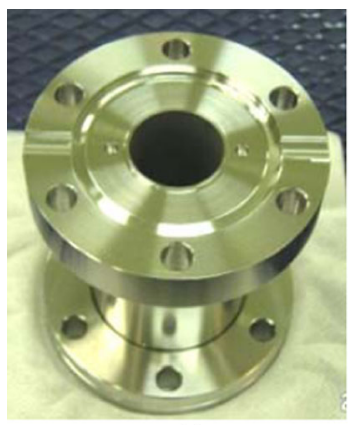

(a)

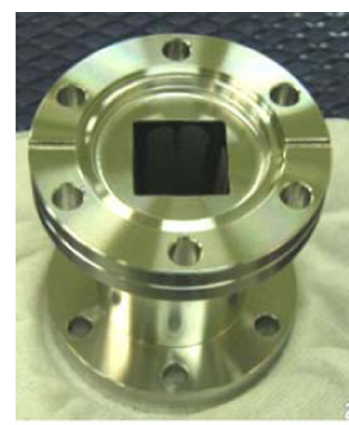

(b)
FIG. 11. Photograph of the beam pipe adaptor. (a) Beam pipe side with circular cross section. (b) Monitor side with square cross section. 


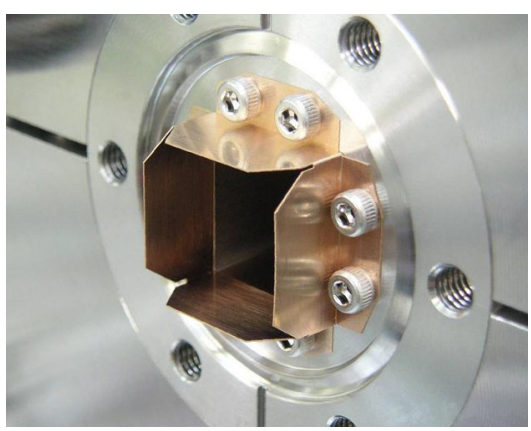

FIG. 12. Photograph of the flange gap finger.

\section{Microstripline structure}

The pulse signal originating from the ionization by induced electrons typically had a length of $0.33 \mathrm{~ns}$ FWHM [7]. The waveform of the pulse would be deformed if an open-wire line was used in the vacuum chamber of the monitor. Therefore, we applied a microstripline structure [9] in the vacuum chamber to improve the high-frequency property (Fig. 13). The microstripline structure is composed of a strip conductor $(\mathrm{Cu})$, a dielectric substrate (ceramic), and a ground plate (stainless steel). The width of the strip conductor and the thickness of the dielectric substrate were designed to give an impedance of $50 \Omega$. High-frequency SMA feedthrough connectors [12], fabricated by Kyocera Corporation, are used to prevent the generation of ringing between the SMA feedthrough connector and the diamond-based detectors. The SMA feedthrough connector has a cutoff frequency of more than $10 \mathrm{GHz}[12,13]$, which satisfies the requirements of the monitor. A demonstration of the transmission of a pulse having a length of 200 ps FWHM using the microstripline structure and the SMA feedthrough connector has been described previously [13].

\section{Diamond-based detectors}

The diamond-based detectors were newly designed [9], as shown in Fig. 14. The diamond crystal was miniaturized from the prototype to adopt the microstripline structure

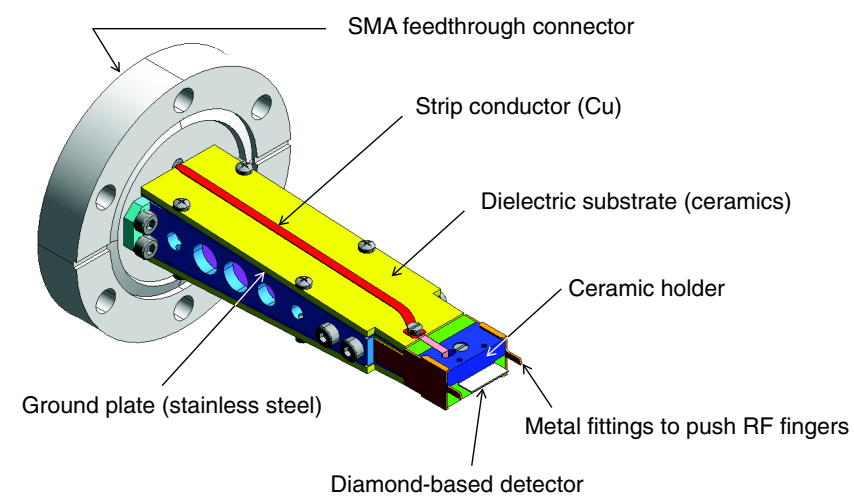

FIG. 13. Microstripline structure with SMA feedthrough connectors on an ICF70 flange. The diamond-based detector is clamped on a ceramic holder.

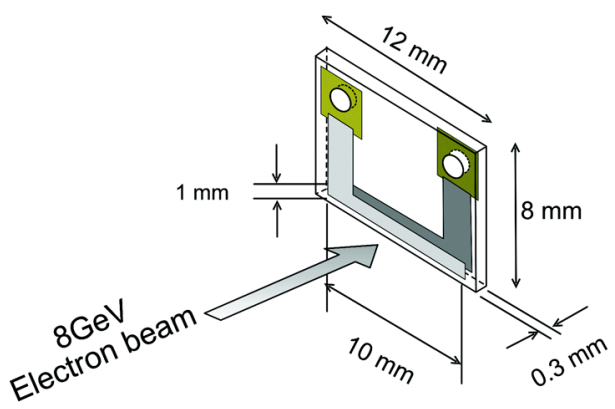

FIG. 14. Dimensions of newly designed diamond-based detector.

and rf fingers easily. It was $12 \mathrm{~mm}(\mathrm{H}) \times 8 \mathrm{~mm}(\mathrm{~V})$ with a thickness of $0.3 \mathrm{~mm}$. The size of the active area, which was sandwiched between electrodes, was $1 \mathrm{~mm}(\mathrm{H}) \times$ $10 \mathrm{~mm}(\mathrm{~W})$ with a thickness of $0.3 \mathrm{~mm}$. The height of the active layer was chosen to be $1 \mathrm{~mm}$. By having this narrow active area, the monitor can be also used for the profile measurement of a beam halo.

We prepared three new diamond-based detectors and evaluated them with $8 \mathrm{GeV}$ electrons from the SPring-8 booster synchrotron [9]. Figure 15 shows the linearity of the charge signal of the detector as a function of the number of incident electrons. We confirmed that all three detectors have good linearity and the same detection efficiency as that of the prototype. One of the three diamondbased detectors was used for the evaluation of wakefield reduction described in Sec. II A and for the evaluation of radiation from rf fingers described in Sec. II B.

\section{E. Final design of beam halo monitor}

Figure 16 shows the final mechanical design of the beam halo monitor employed in SACLA $[9,10]$. The beam halo

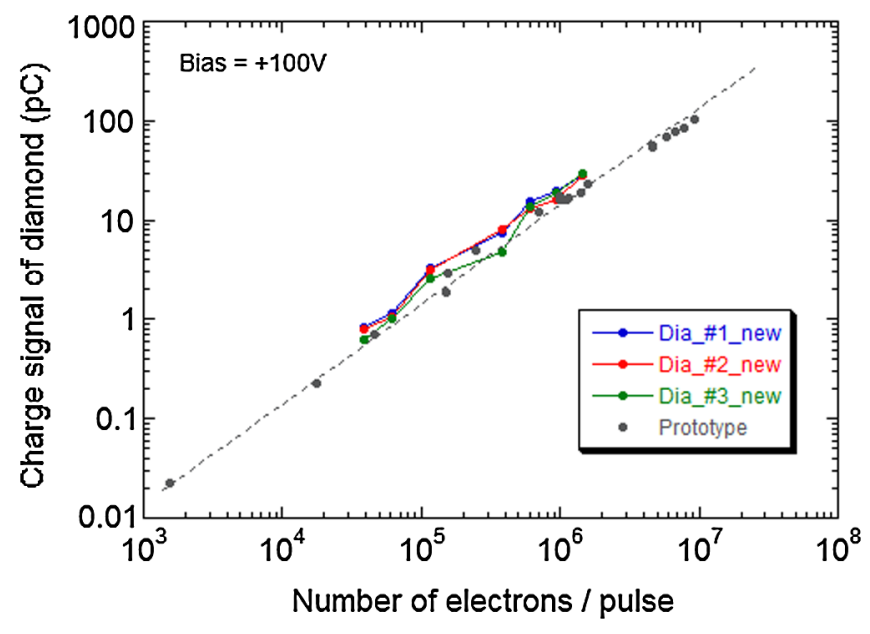

FIG. 15. Linearity of charge signal of the diamond-based detectors as a function of the number of incident electrons. Black dots indicate the results for the prototype diamond-based detector [7]. Typical pulse length of the incident electrons is 50 ps $(\sigma)$. 


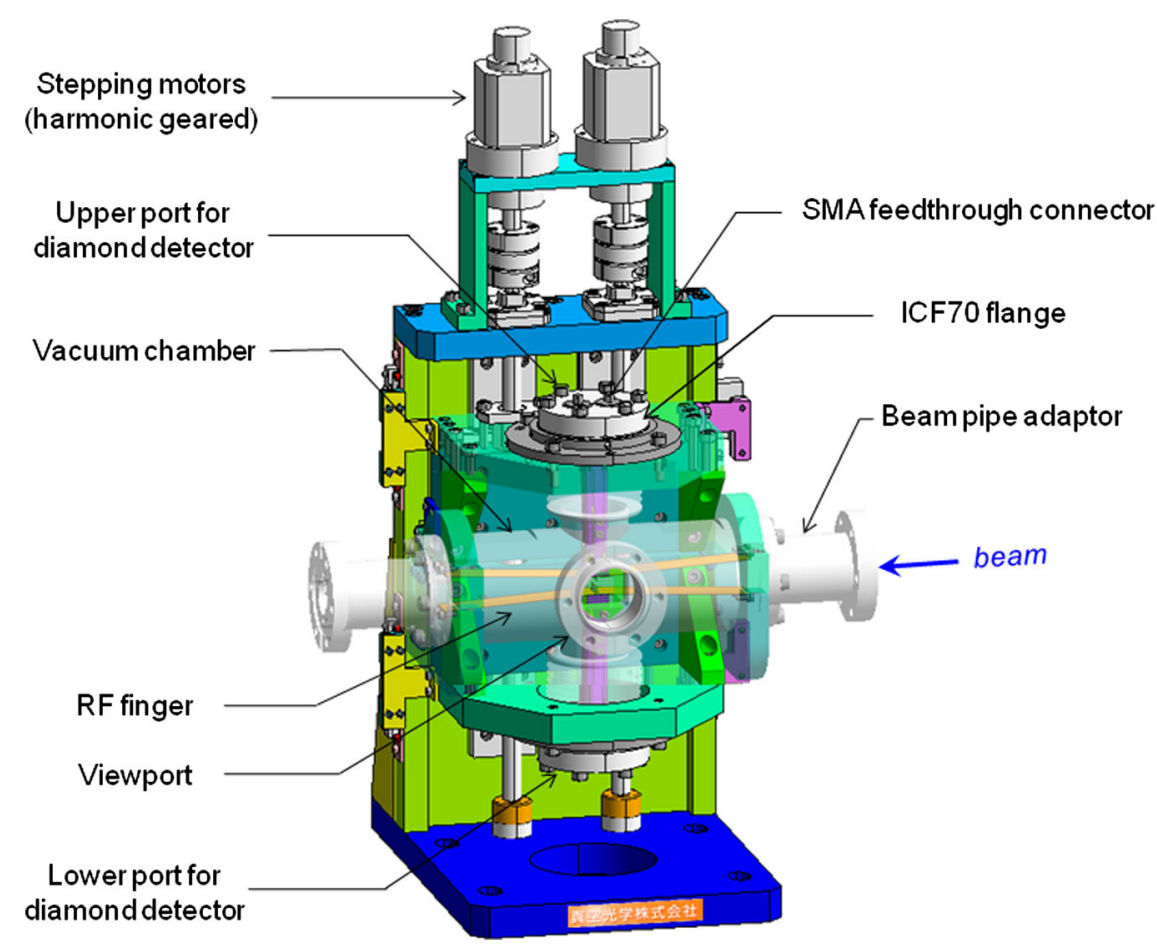

FIG. 16. Illustration of the beam halo monitor employed in SACLA.

monitor is equipped with $\mathrm{rf}$ fingers with $\mathrm{Al}$ windows, which have the type 2 configuration as mentioned earlier. Beam pipe adaptors are connected to the upstream and downstream sides of the vacuum chamber of the monitor. The diamond-based detectors on both the upper and lower ports can be independently manipulated using stepping motors. The vacuum chamber and beam pipe adaptors are fixed during the manipulation of the detectors. Thus, there is no transverse offset that causes mechanical stress on the bellows attached to both beam pipe adaptors. Because the monitor is used under an ultrahigh-vacuum condition, it is designed to be baked in vacuum.

The beam halo monitor is installed about $1 \mathrm{~m}$ upstream of the undulators to monitor the intensity of the beam halo that irradiates the undulator permanent magnets (Fig. 17) [14]. In the area several meters upstream of the undulators, it is necessary to decrease the geomagnetic field of $0.4 \mathrm{G}$ by about 100 -fold for the electron beam to propagate in a straight line in this area. Therefore, the beam halo monitor is covered by a geomagnetic shield box so as to attenuate the magnetic field on the beam axis [15].

As a data acquisition system, the preamplifier prepared for the evaluation of the prototype is combined with an event-synchronized data acquisition system in SACLA. The preamplifier was originally developed for the current transformer (CT) in the SCSS test accelerator [16]. The time constant of the amplifier was adjusted to about $40 \mathrm{~ns}$ to match the event-synchronized data acquisition system and to effectively suppress the ringing noise caused by the wakefield of the electron beam. This system can record

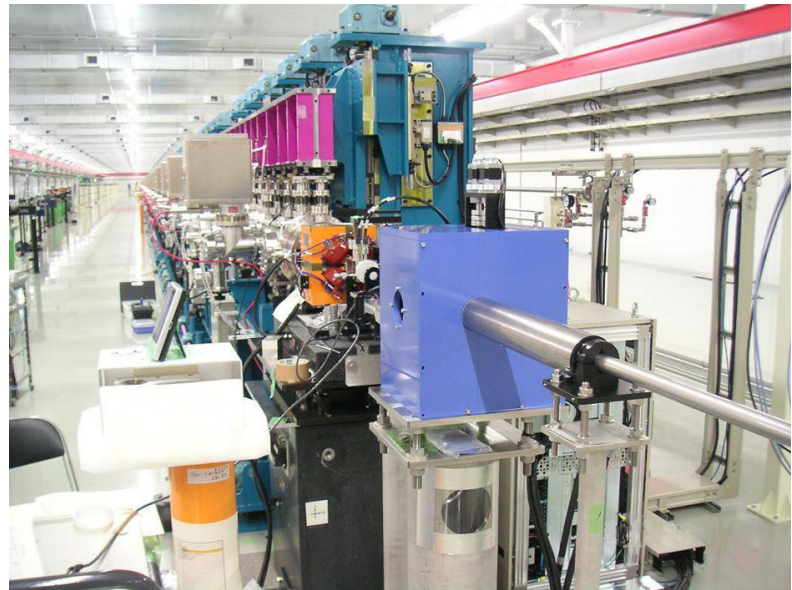

FIG. 17. Photograph of the beam halo monitor covered with the geomagnetic shield box (painted in blue). The large equipment behind the monitor is the set of undulators. The beam direction is from lower right to upper left.

every set of output signals that are synchronized with electron beam shots [17]. We also prepared a system to manipulate the diamond-based detectors from a central control room.

\section{OPERATIONAL RESULTS IN SACLA}

\section{A. Effect on electron beam quality}

In order for the beam halo monitor to be continually used in SACLA, the degradation of the electron beam must 


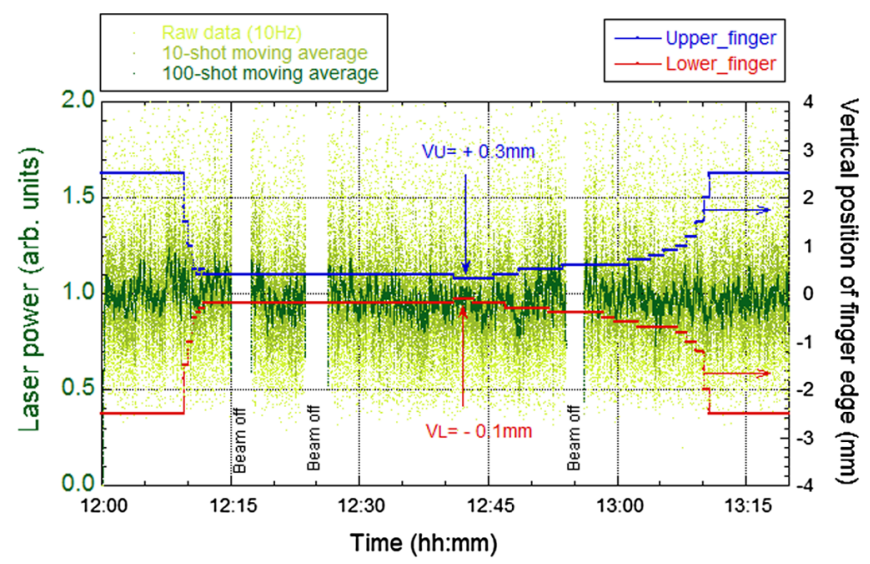

FIG. 18. Behavior of laser power (left axis) and vertical position of edges of rf fingers (right axis) over time. The minimum gap is $0.4 \mathrm{~mm}$ at approximately $12: 42$.

be avoided, even when the diamond detectors covered with the rf fingers approach the beam axis. Figure 18 shows the behavior of the laser power when the distance from the edges of the rf fingers to the beam axis is changed [14]. The laser power does not change even when the gap between the rf fingers is $0.4 \mathrm{~mm}$, where the vertical position of the upper (lower) finger edge $V_{U}\left(V_{L}\right)$ is at $+0.3 \mathrm{~mm}$ $(-0.1 \mathrm{~mm})$. This result means either that the rf shielding is very efficient or the geometrical wakefields are not greatly damaging the bunch energy distribution.

\section{B. Filtering of residual high-frequency components with LPFs}

We adopt LPFs to suppress the effect of residual high-frequency components [9], which originate from the induction current of the beam core. Figure 19 shows the effect of the residual high-frequency components before and after filtering. The cutoff frequency of the LPFs was set at $117 \mathrm{MHz}$, which is the same as that in the prototype [7]. In the case that the original current signal originating from ionization by induced electrons is larger than the residual high-frequency components, one can clearly observe that the residual high-frequency components are filtered and the original current signal is enhanced, as shown in Figs. 19(a) and 19(b). In the case that the original current signal is very small compared with the residual highfrequency components, one can also observe that the original current signal is enhanced and the residual high-frequency components are filtered, as shown in Figs. 19(c) and 19(d). We conclude that the reduction in the intensity of high-frequency components becomes possible by adopting the rf fingers with aluminum windows and that the residual high-frequency components are efficiently suppressed by the LPFs.

\section{Observation of scattering of beam core at $\mathbf{r f}$ fingers}

The rf fingers with aluminum windows were adopted for the beam halo monitor employed in SACLA. The fingers expanded by $1 \mathrm{~mm}$ from the detector tip toward the beam (a)

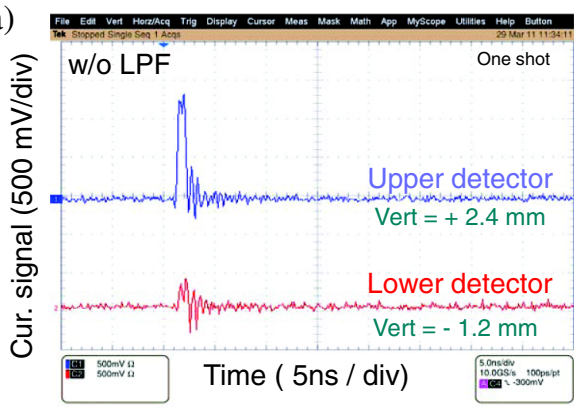

(c)

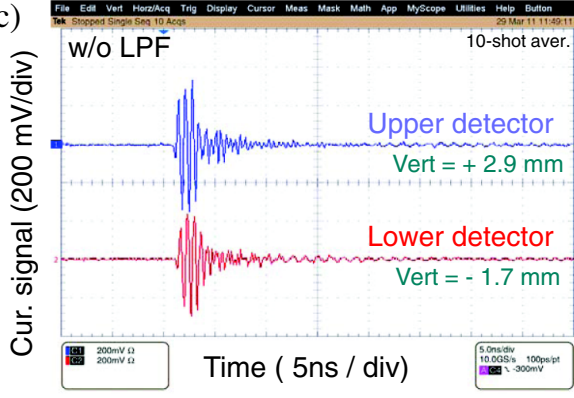

(b)

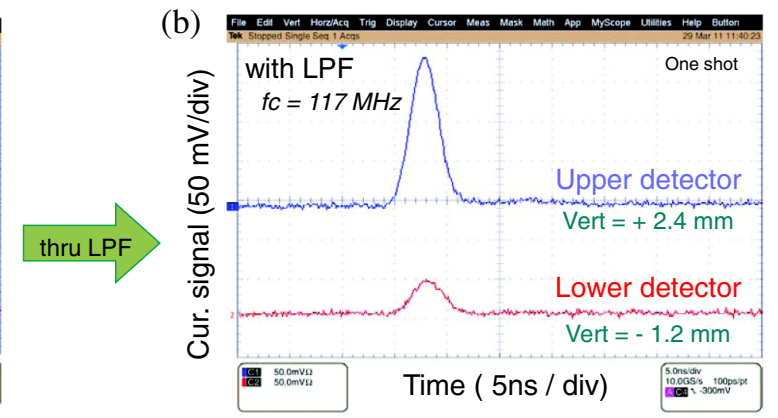

(d)

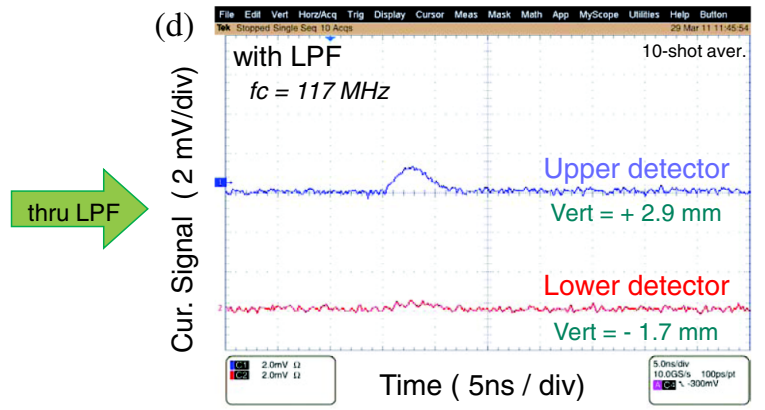

FIG. 19. Filtering of residual high-frequency components without (w/o) or with LPFs. (a) Large original current signal w/o LPFs, (b) large original current signal with LPFs, (c) small original current signal w/o LPFs, and (d) small original current signal with LPFs. 
core. When the edges of the rf fingers are irradiated by the beam core, scattering radiation is generated, resulting in an increase in the output signal of the diamond detectors. To evaluate this effect, we observed the output signal of the diamond detectors when either of the upper or lower diamond detectors was moved from the position at which the gap between the upper and lower rf fingers was $0.4 \mathrm{~mm}$ (Fig. 20) [14]. L1 and L2 (U1 and U2) indicate that only the lower (upper) finger was moved. The output signal of the upper (lower) detector also changed at L1 and L2 (U1 and U2). This result suggests that the core of the beam is scattered at the edge of the rf fingers.

Figure 21 shows the charge signal of the diamond-based detectors versus the vertical position of the edge of the $\mathrm{rf}$ fingers. The solid lines indicate the data measured after the XFEL lasing of SACLA. When the edges of the rf fingers are near the beam core, the charge signals of the diamond detectors increase in intensity because of scattering. On the other hand, when the absolute value of the vertical position of the rf finger edge is $1 \mathrm{~mm}$ or higher, the charge signal decreases in intensity to below the lower detection limit of $2 \times 10^{3}$ electrons/pulse [7]. Therefore, we conclude that a charge signal originating from a beam halo with a distance from the beam axis of $2 \mathrm{~mm}$ or more is below the lower detection limit. In other words, there are no effects of the dark current, secondary electrons, or bremsstrahlung generated in the accelerator on the charge signal. This result means that the permanent magnets of the undulators are not demagnetized by the irradiation of the beam halo when the gap between the undulator magnets is more than $4 \mathrm{~mm}$.

In Fig. 21, the following features can also be observed. The dashed lines indicate the data measured during the early stage of the commissioning of the linear accelerator of SACLA. The effect of scattering at the edge of the rf fingers can be seen in a larger area than that after XFEL lasing [14]. This observation suggests that the size of the

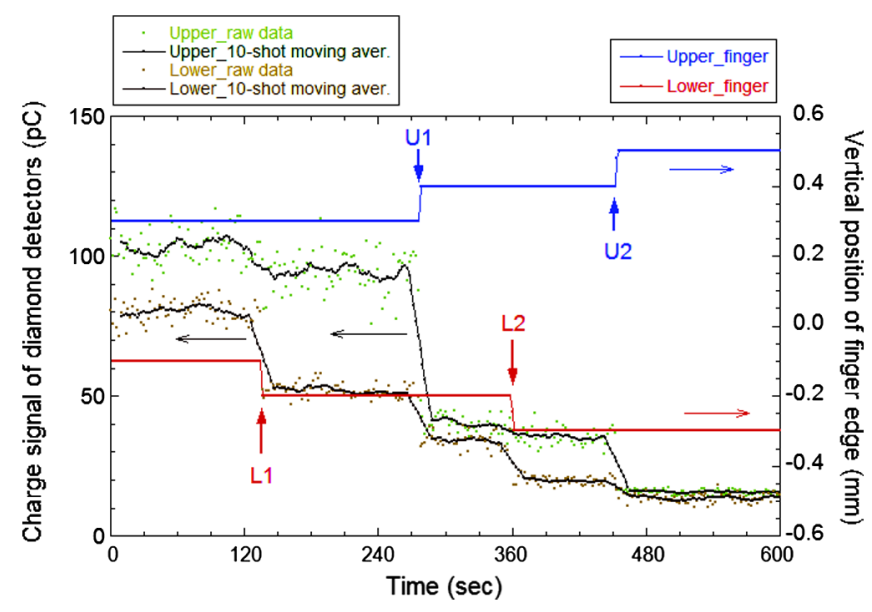

FIG. 20. Behavior of charge signal of diamond-based detectors when the vertical position of the finger edge is changed. The gap was varied from 0.4 to $0.8 \mathrm{~mm}$.

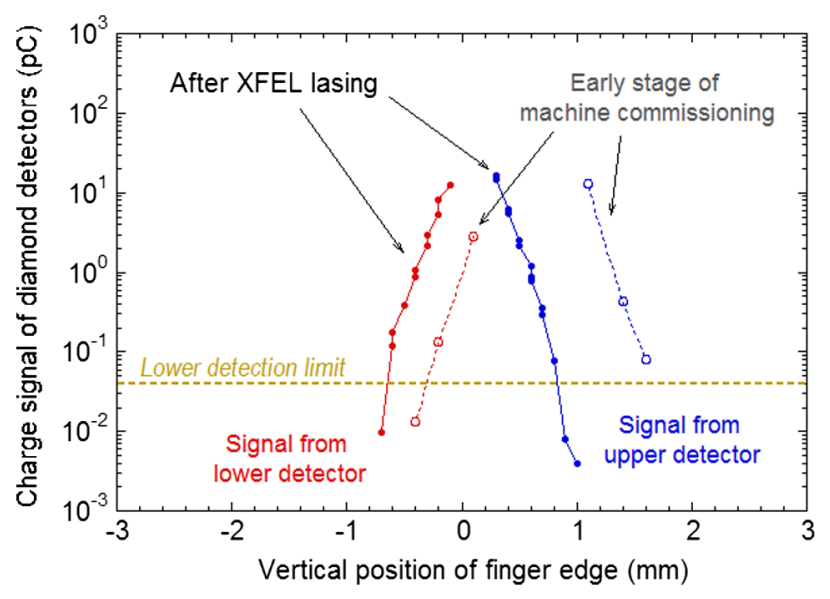

FIG. 21. Effect of rf finger edge position on charge signal of diamond-based detectors. Solid lines were obtained after XFEL lasing. Dashed lines were obtained during the early stage of machine commissioning. The lower detection limit of $2 \times 10^{3}$ electrons/pulse, which corresponds to $0.04 \mathrm{pC}$, is indicated. The typical beam size after XFEL lasing is $0.06 \mathrm{~mm}(\sigma)$. The beam size during the early stage of machine commissioning is estimated to be much larger than that.

beam core decreased during the commissioning, and that the position of the beam core approached the center. In this way, the beam halo monitor can be used as a beam diagnostic tool during the accelerator commissioning as well as during regular accelerator tuning.

\section{CONCLUSIONS}

The electron beam halo monitor employed in SACLA, which has rf fingers with $\mathrm{Al}$ windows, was newly designed and fabricated. The beam halo monitor was installed upstream of the undulators to prevent the demagnetization of the permanent magnets of the undulators by the irradiation of the beam halo.

The rf fingers with $\mathrm{Al}$ windows were designed to fully cover the diamond-based detectors. The merits of this configuration are as follows: (1) it removes the wakefield, thus preserving the electron beam quality and stabilizing XFEL lasing, and (2) it reduces the intensity of highfrequency components that emerge in the output signal originating from the ionization by induced electrons on diamond-based detectors. We evaluated the effect of radiation from the $\mathrm{Al}$ windows on the output signal both experimentally and by simulation, and confirmed that the effect is negligible under our conditions of use.

To further improve the high-frequency properties, (a) other rf fingers, such as a screen shield, beam pipe adaptors, and flange gap fingers, have been added to the vacuum chamber of the monitor, (b) a microstripline structure has been installed in the vacuum chamber, and (c) the diamond detectors have been miniaturized.

We demonstrated the performance of the beam halo monitor during the commissioning of SACLA. We found 
that the laser power is not affected by the rf fingers, the intensity of high-frequency components can be reduced by adopting the rf fingers with aluminum windows, and the residual high-frequency components are efficiently filtered by LPFs. Profile measurements suggest that the beam size decreased as the commissioning advanced. As a next step, we consider that the rf fingers can be improved such that the edge of the rf fingers will not be irradiated by the beam core.

During the user operation of SACLA, the intensity of the beam halo is continually monitored using a data acquisition system. We found that the intensity of the beam halo is always below the detection limit.

\section{ACKNOWLEDGMENTS}

The authors would like to thank the SCSS Test Accelerator Operation Group and EUV-FEL Experimental Facility Team of RIKEN/SPring-8 for their support in the beam tests. The authors would also like to thank the SACLA Beam Dynamics Team for their comments and support during the start-up tuning of the beam halo monitor. The preparation of the experimental hardware was supported by S. Takahashi and A. Watanabe of the Light Source and Optics Division, JASRI/SPring-8. Useful advice was given by S. Inoue, T. Ohshima, H. Maesaka, and Y. Otake of the SACLA Accelerator Development Team. The diamond-based detectors were fabricated by Kobe Steel, Ltd. The beam halo monitors and rf fingers were fabricated by Vacuum and Optical Instruments (Shinku-Kogaku, Inc). This work was partly supported by Japan Society for the Promotion of Science through a Grant-in-Aid for Scientific Research (c), No. 21604017.

[1] T. Bizen et al., Nucl. Instrum. Methods Phys. Res., Sect. A 574, 401 (2007).

[2] T. Behnke, M. Doucet, N. Ghodbane, A. Imhof, C. Martınez, and W. Zeuner, Nucl. Instrum. Methods Phys. Res., Sect. A, 489, 230 (2002).

[3] R. J. Tapper, Rep. Prog. Phys. 63, 1273 (2000).

[4] L. Fernandez-Hernando, D. Chong, R. Gray, C. Ilgner, A. Macpherson, A. Oh, T. Pritchard, R. Stone, and S. Worm, Nucl. Instrum. Methods Phys. Res., Sect. A 552, 183 (2005).

[5] E. Griesmayer, B. Dehning, E. Effinger, and D. Dobos, in Proceedings of the 14th Beam Instrumentation Workshop,
Santa Fe, New Mexico (LANL, Los Alamos, 2010), MOCNB02.

[6] H. Aoyagi, T. Kudo, H. Tanida, and H. Kitamura, in Proceedings of the 8th International Conference on Synchrotron Radiation Instrumentation, San Francisco, California, AIP Conf. Proc. No. 705 (AIP, New York, 2004), p. 933.

[7] H. Aoyagi, Y. Asano, T. Itoga, N. Nariyama, T. Bizen, T. Tanaka, and H. Kitamura, Phys. Rev. ST Accel. Beams 15, 022801 (2012).

[8] X.-M. Marechal, Y. Asano, and T. Itoga, Nucl. Instrum. Methods Phys. Res., Sect. A 673, 32 (2012).

[9] H. Aoyagi, T. Bizen, N. Nariyama, Y. Asano, T. Itoga, H. Kitamura, and T. Tanaka, in Proceedings of the 32nd Free Electron Laser Conference, Malmö, Sweden (Max-lab, Sweden, 2010), THOC4.

[10] H. Aoyagi, T. Bizen, N. Nariyama, S. Suzuki, K. Fukami, T. Aoki, Y. Asano, T. Itoga, H. Kitamura, and T. Tanaka, in Proceedings of the 10th European Workshop on Beam Diagnostics and Instrumentation for Particle Accelerators, Hamburg, Germany (DESY, Hamburg, 2011), MOOB03.

[11] http://rcwww.kek.jp/research/egs/.

[12] H. Aoyagi, T. Kudo, T. Matsushita, S. Takahashi, K. Iwamoto, T. Kitamura, and H. Kitamura, in Proceedings of the 9th International Conference on Synchrotron Radiation Instrumentation, Daegu, Korea, AIP Conf. Proc. No. 879 (AIP, New York, 2007), p. 1010.

[13] H. Aoyagi, S. Takahashi, and H. Kitamura, in Proceedings of the 10th European Workshop on Beam Diagnostics and Instrumentation for Particle Accelerators, Hamburg, Germany (DESY, Hamburg, 2011), MOPD91.

[14] H. Aoyagi, Y. Asano, H. Kitamura, and T. Tanaka, in Proceedings of the 2nd International Particle Accelerator Conference, San Sebastián, Spain (EPS-AG, Spain, 2011), WEPEB068.

[15] H. Aoyagi, T. Hasegawa, T. Bizen, and Y. Asano, in Proceedings of the 8th Annual Meeting of the Particle Accelerator Society of Japan, Tsukuba, Japan (PASJ, Japan, 2011), TUPS161.

[16] A. Higashiya, H. Maesaka, and Y. Otake, in Proceedings of the 29th Free Electron Laser Conference, Novosibirsk, Russia (BINP, Novosibirsk, 2007), WEPPH051.

[17] M. Yamaga, Y. Furukawa, T. Hirono, M. Ishii, T. Masuda, T. Ohata, R. Tanaka, A. Yamashita, T. Fukui, and N. Hosoda, in Proceedings of the 12th International Conference on Accelerator and Large Experimental Physics Control Systems, Kobe, Japan (SPring-8, Japan, 2009), TUB003. 